\title{
Expressions of miR-30c and let-7a are inversely correlated with HMGA2 expression in squamous cell carcinoma of the vulva
}

\author{
Antonio Agostini ${ }^{1,2}$, Marta Brunetti ${ }^{1,2}$, Ben Davidson ${ }^{3,4}$, Claes G. Trope ${ }^{5}$, Sverre \\ Heim $^{1,2,4}$, Ioannis Panagopoulos ${ }^{1,2}$, Francesca Micci, ${ }^{1,2}$ \\ ${ }^{1}$ Section for Cancer Cytogenetics, Institute for Cancer Genetics and Informatics, The Norwegian Radium Hospital, Oslo \\ University Hospital, Oslo, Norway \\ ${ }^{2}$ Centre for Cancer Biomedicine, University of Oslo, Oslo, Norway \\ ${ }^{3}$ Department of Pathology, The Norwegian Radium Hospital, Oslo University Hospital, Oslo, Norway \\ ${ }^{4}$ Faculty of Medicine, University of Oslo, Oslo, Norway \\ ${ }^{5}$ Department of Gynecology, The Norwegian Radium Hospital, Oslo University Hospital, Oslo, Norway \\ Correspondence to: Francesca Micci, email: francesca.micci@labmed.vio.no \\ Keywords: HMGA2, miR-30c, let-7a, FHIT
}

Received: September 14, $2016 \quad$ Accepted: October 22, $2016 \quad$ Published: November 07, 2016

\section{ABSTRACT}

Malignant tumors of the vulva, most of them squamous cell carcinomas, account for only $5 \%$ of cancers of the female genital tract. Though little is known about the genetic features of these tumors, the Fragile Histidine Triad (FHIT) and High Mobility Group AT-hook 2 (HMGA2) genes were found deregulated. We wanted to gain more knowledge about the expression of HMGA2-related miRNAs such as miR$30 \mathrm{c}$ and let-7a, and whether a correlation exists between the expression of FHIT and HMGA2, in this tumor type. An inverse correlation was found in-as-much as HMGA2 was highly expressed (mean fold change 8.8) whereas miR30c and let-7a were both downregulated (mean fold change -3.9 and -2.3, respectively). The consistent overexpression of HMGA2 found in all tumors adds to the likelihood that this gene is of importance in SCC pathogenesis. Moreover, we came to the conclusion that miRNAs may be the cause of the deregulation of HMGA2. Our results also show that SCC of the vulva presents a characteristic molecular pattern with FHIT being downregulated whereas HMGA2 is upregulated.

\section{INTRODUCTION}

Squamous cell carcinomas (SCC) account for $70 \%$ of all malignant tumors arising in the vulva. [1]. Little is known about the genetic features of this cancer as only 44 cases of vulvar SCC have been cytogenetically and/ or molecularly analyzed [2-6]. Our group has previously reported a chromosome- and array-based comparative genomic hybridization (CGH) analysis of vulvar SCC which showed, among other imbalances, frequent loss of chromosomal band 3p14 [2]. The Fragile Histidine Triad (FHIT) tumor suppressor gene, which maps to this band, was found downregulated [2]. Recently, we showed expression of the High mobility group AT-hook 2 gene (HMGA2) in $86 \%$ (20 out of 23 ) of vulvar SCC analyzed [7].
An inverse correlation between the expression of FHIT, miR-30c, and HMGA2 was found by Suh et al. [6] in lung cancer. FHIT enhances the expression of miR-30c as well as $H M G A 2$ repression thereby inhibiting epithelialmesenchymal transition and the metastatic process in small cell carcinoma of the lung. The interaction between this miRNA and the HMGA2 3'untranslated region

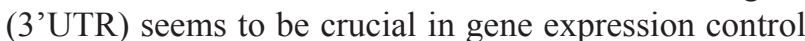
[8]. Since our previous findings in SCC of the vulva [2] hint at a similar expression correlation as that detected in lung cancer, we decided to quantify the expression levels of $H M G A 2$ and miR-30c in the 10 tumors of the vulva that were found to have deregulated HMGA2 and FHIT, and to assess the expression levels of the miRNA let-7a which is known to target and repress $H M G A 2$ [9-11], as well as the expression of the let-7a regulators LIN28A and LIN28B [12]. 
Table 1: Overview of the samples and results

\begin{tabular}{|c|c|c|c|c|c|c|c|}
\hline Case/Lab Number & Diagnosis $^{\mathrm{a}}$ & FHIT & miR-30c & LIN28A & LIN28B $^{d}$ & Let-7a & HMGA2 \\
\hline $1 / 02-167$ & $\mathrm{SCC} \mathrm{m}$ & $\downarrow$ & $\downarrow$ & $\downarrow$ & - & $\downarrow$ & $\uparrow$ \\
\hline $2 / 02-848$ & $\mathrm{SCC} \mathrm{m}$ & $\downarrow$ & $\downarrow$ & $\downarrow$ & - & $\downarrow$ & $\uparrow$ \\
\hline $3 / 02-869$ & $\mathrm{SCC} h, \mathrm{~m}, \mathrm{p}$ & $\downarrow$ & $\downarrow$ & $\downarrow$ & - & $\downarrow$ & $\uparrow$ \\
\hline $4 / 02-1171$ & $\mathrm{SCC}$ & $\downarrow$ & $\downarrow$ & $\uparrow$ & - & $\downarrow$ & $\uparrow$ \\
\hline $5 / 03-830$ & $\mathrm{SCC} \mathrm{m}$ & $\downarrow$ & $\downarrow$ & $n e^{c}$ & - & $\downarrow$ & $\uparrow$ \\
\hline 6/03-1011 & $\mathrm{SCC} h, \mathrm{~m}$ & $\uparrow$ & $\downarrow$ & $n e^{c}$ & - & $\downarrow$ & $\uparrow$ \\
\hline 7/03-1088 & $\mathrm{SCC} \mathrm{h}$ & $\downarrow$ loss $3 \mathrm{p} 14^{\mathrm{b} *}$ & $\downarrow$ & $\uparrow$ & - & $\downarrow$ & $\uparrow$ \\
\hline 8/06-19 & $\mathrm{SCC} p$ & $\downarrow$ & $\downarrow$ & $n e^{c}$ & - & $\downarrow$ & $\uparrow$ \\
\hline $9 / 06-125$ & $\mathrm{SCC} \mathrm{m}$ & $\downarrow \operatorname{loss} 3 \mathrm{p} 14^{\mathrm{b}}$ & $\downarrow$ & $\uparrow$ & - & $\downarrow$ & $\uparrow$ \\
\hline $10 / 09-733$ & $\mathrm{SCC} h$ & $\downarrow$ loss $3 \mathrm{p} 14^{\mathrm{b}}$ & $\downarrow$ & $\downarrow$ & - & $\downarrow$ & $\uparrow$ \\
\hline
\end{tabular}

Arrows are used to show deregulation of the genes (arrow head up: up regulation; arrow head down: down regulation) ${ }^{a} \mathrm{~h}$ : highly differentiated, m: moderately differentiated, p: poorly differentiated.

${ }^{\mathrm{b}}$ Genomic Imbalances assessed with an array based Comparative Genomic Hybridization (aCGH) showing a loss of the FHIT locus. * aCGH detected a homozigous deletion for the FHIT locus.

${ }^{c}$ ne: normal expression (no differences between samples and the normal controls expression levels).

${ }^{\mathrm{d}}$ No LIN28B expression was found in our samples.

\section{RESULTS}

All molecular investigations gave informative results (Table 1) which were normalized using the two normal vulva tissue samples. HMGA2 was expressed at high levels in all 10 tumors analyzed with an 8.8 fold change average (range: 7-14) (Figure 1A). miR-30c was strongly downregulated in all 10 tumors (Figure 1B) with an average fold change of -3.9 (range: -2.3 to -5.5 ). The analysis of let-7a expression found downregulation in all cases with a mean -2.3 fold change (range: -0.5 to -3.5 ) (Figure 1). LIN28A was found deregulated in four out of ten tumors. In these samples, the detected fold changes were -1.5 for cases 1 and $2,-3.5$ for case 3 , and -1 for case 10 with a mean fold change of -1.8 . Three tumors (cases 4, 7, and 9) showed upregulation of LIN28A with a fold change of 2.5 for case 4,6 for case 7 , and 1.5 for case 9 giving a mean fold change of 3.3. In the remaining three tumors (cases 5, 6, and 8), LIN28A expression was similar to that of the normal controls (Figure 1A). No expression of $L I N 28 B$ was detected in the 10 SCC of the vulva or in the two normal controls. However, the gene was found expressed in the Total Human Universal Reference used as an internal control for each reaction.

Searching for possible causes behind the deregulation of let-7a and miR-30c in vulvar SCC, we checked the genomic imbalance charts of these tumors to find if the miRNA clusters for let-7a and miR-30c were deleted. These data were retrived from our previous publication on the very same samples in which an array Comparative Genomic Hybridization study was described
[2], looking specifically for the three genes expressing let-7a, MIRLET7A1 on 9q22.3, MIRLET7A2 on 11q24.1, and MIRLET7A3 on 22q13.3, and the two genes for miR30c, MIR30C1 and MIR30C2, mapping on $1 \mathrm{p} 34.2$ and $6 q 13$, respectively. No genomic loss was found for the chromosomal bands in which these miRNAs clusters are located, the only exception being case 10 , where a deletion was found in the chromosome arms containing all three MIRLET7A genes (6q, 9q, and 22q).

\section{DISCUSSION}

Few SCC of the vulva have been characterized both at the cytogenetic and molecular level. HMGA2 is one of the few genes found to be activated in this tumor type [6]; however, no previous study has quantified its expression. We found that $H M G A 2$ was expressed at high levels in all 10 vulvar SCC analyzed with an average fold change of 8.8. HMGA2 belongs to the High-mobility group AT-hook family of non-histone proteins involved in a wide variety of cellular processes from DNA damage repair to gene regulation [13]. The gene is expressed during embryonic development [14] but is usually unexpressed in adult normal tissues [15]. High expression levels of $H M G A 2$ have been found in various types of tumors including benign connective tissue neoplasms and malignant tumors [16]. HMGA2 promotes diverse tumorigenic processes from cellular proliferation to epithelial-mesenchymal transition (EMT) and metastasis formation [17]. The mechanisms that lead to unchecked expression of $H M G A 2$ in cancer are still 
not fully understood, but miRNA-dependent regulation seems to play a role. The HMGA2 3'untranslated region (3'UTR) contains regulatory sequences that are targeted by different families of miRNAs [8], and miRNA-dependent repression seems to be the main mechanism to ensure negative $H M G A 2$ expression [10, 11, 18]. Another piece of evidence testifying to the importance of the interaction between HMGA2 and miRNAs is the high frequency of disrupted forms of this gene, due to rearrangement of chromosomal band 12q15, found in different tumors [19]. These alterations bring about loss of the 3'UTR leading to a truncated transcript that evades miRNA-dependent gene silencing [19]. Interestingly, no truncated form of the gene was found in the investigated samples [6], further hinting at the importance of alternative mechanisms of gene deregulation in vulvar SCC. To test this further, we decided to assess the expression of two miRNAs, miR-30c and let$7 \mathrm{a}$, that are known to target and regulate HMGA2 [9, 18].
Downregulation of miR-30c was found in all cases analyzed. For the first time in SCC of the vulva, we showed an inverse correlation betrween miR-30c and $H M G A 2$ expression similar to the one previously reported in lung cancer [18]. Moreover, when retrieving data on FHIT expression from our previous publication [2], we saw similar downregulation of FHIT and miR$30 \mathrm{c}$, admittedly with one sample (case 6) showing a high expression of FHIT in spite of miR-30c downregulation. This indicates that even other causes of miR-30c downregulation may exist in vulvar SCC.

We found downregulation of let-7a in all tumors analyzed. Deregulation of the let-7 family of miRNAs occurs in different types of tumors and the most important result of it seems to be $H M G A 2$ overexpression [20, 21]. This is also the case for SCC of the vulva, where deregulation of $H M G A 2$ could be brought on by altered expression of FHIT, miR-30c, and/or let-7a.
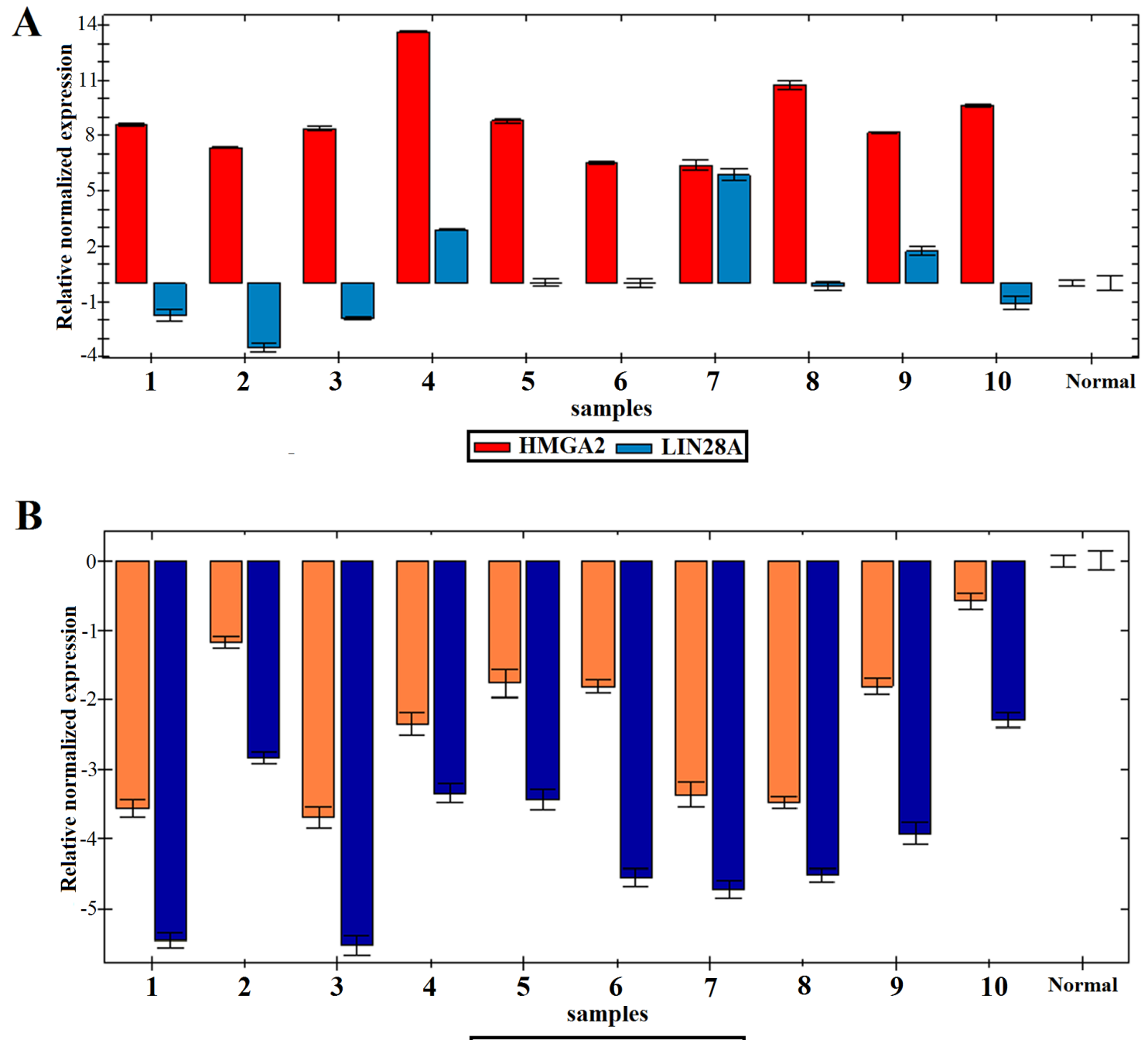

Figure 1: Expression profile of the 10 SCC of the vulva. A. Relative normalized expression of $H M G A 2$ and $L I N 28 A$. B. Relative normalized expression of miR-30c and let-7a. 
Allegedly, deregulation of the let-7 family of miRNA is caused by overexpression of the RNA-binding protein homologues LIN28A and LIN28B that inhibit the maturation of both pri-let-7 and pre-let-7 [12]. Since only three out of ten tumors had overexpression of LIN28A, we conclude that LIN28A is not the primary cause of let-7a downregulation in SCC of the vulva. The correlation between let-7a downregulation and HMGA2 overexpression was previously found also in SCC of the oral cavity [22] and esophagus [23]. These findings strengthen our interpretation and lead us to assume that miRNA deregulation is the major cause of the high $H M G A 2$ expression levels in SCC of the vulva, possibly in squamous cell carcinomas generally.

Our study showed that SCC of the vulva presents a characteristic molecular signature in that FHIT is downregulated while $H M G A 2$ is upregulated. The deregulation of these genes may be correlated with the invasive capacity of these tumors as shown in lung cancer [18]. FHIT is a suppressor gene with a role in apoptosis and epithelial-mesenchymal transition (EMT) [24, 25]. HMGA2 induces EMT and invasiveness in epithelial tumors and high expression levels of the gene have been seen in primary as well as metastatic carcinomas [26]. HMGA2 enhances the TGF $\beta$-SMADs signaling pathway [27] and so upregulates the major transcription factors involved in EMT, i.e, SNAIL1, SNAIL2, and TWIST. The consistent overexpression of HMGA2 found in 10 out of 10 tumors adds to the likelihood that this gene is of general importance in SCC pathogenesis. MiRNAs may be the cause of the HMGA2 deregulation. This irrespective, the deregulation of these genes together with downregulation of FHIT constitutes a molecular signature of vulvar SCC.

\section{MATERIALS AND METHODS}

\section{Tumor material}

The material consisted of fresh frozen samples from 10 SCC of the vulva and two normal vulva specimens surgically removed at The Norwegian Radium Hospital (Table 1). The tumors have previously been examined for chromosomal aberrations and genomic imbalances as well as for expression of FHIT [2, 4] and HMGA2 [6]. The study was approved by the regional ethics committee (Regional komité for medisinsk forskningsetikk Sør-Øst, Norge, http://helseforskning.etikkom.no) and written informed consent was obtained from the patients.

\section{Total RNA extraction}

Total RNA was extracted using the miRNeasy Kit (Qiagen, Hilden, Germany) and QIAcube (Qiagen) according to the manufacturers' recommendations. RNA concentration and purity was measured using a Nanovue Spectrophotometer (GE Healthcare, Pittsburgh, PA, USA).

\section{Real-time polymerase chain reaction (real-time PCR)}

The expression of the target miRNAs and genes was assessed with Real-Time PCR. The PCR analyses were performed using the CFX96 Touch Real-Time PCR detection system (Bio-Rad Laboratories, Oslo, Norway). The reactions were carried out in quadruplicate using the TaqMan Universal Master Mix II with UNG (Applied Biosystems, Foster City, CA, USA) following the manufacturer's protocol. Human Universal Reference Total RNA (Clontech, Mountain View, CA, USA) was used as internal reaction control with two samples of normal vulva tissue being used for normalization.

\section{MicroRNA expression}

Ten ng of total RNA were reverse transcribed with the TaqMan MicroRNA Reverse Transcription Kit (Applied Biosystems) following the manufacturer's protocol. miRNA expression was assessed with RealTime PCR using the TaqMan MicroRNA Assays (Applied Biosystems) for let-7a (TM:000377) and miR-30c (TM:000419). The RNU6B gene (TM:001093) was used as a reference.

\section{Gene expression}

One $\mu \mathrm{g}$ of extracted total RNA was reversetranscribed in a $20 \mu$ reaction volume using iScript Advanced cDNA Synthesis Kit according to the manufacturer's instructions (Bio-Rad Laboratories, Oslo, Norway). Gene expression was assessed with Real-Time PCR using the TaqMan Gene Expression Assays (Applied Biosystems): HMGA2 (Hs_04397751_m1), FHIT (Hs_00179987_m1), LIN28A (Hs_00702808_s1), LIN28B (Hs_01013729_m1), and ACTB (Hs_01939407_gH). The latter gene was used as a reference.

\section{ACKNOWLEDGMENTS}

This work was supported by grants from the Norwegian Radium Hospital Foundation.

\section{COMPETING INTEREST}

The authors have no competing interest to disclose.

\section{REFERENCES}

1. Wilkinson E. (2011). Premalignant and Malignant Tumors of the Vulva. In: Kurman R, Ellenson L and Ronnett B, eds. Blausteins Pathology of the Female Genital Tract: Springer US), pp. 55-103. 
2. Micci F, Panagopoulos I, Haugom L, Dahlback HS, Pretorius ME, Davidson B, Abeler VM, Trope CG, Danielsen HE, Heim S. Genomic aberration patterns and expression profiles of squamous cell carcinomas of the vulva. Genes ChromosomesCancer. 2013; 52:551-563.

3. Teixeira MR, Kristensen GB, Abeler VM, Heim S. Karyotypic findings in tumors of the vulva and vagina. Cancer Genet Cytogenet. 1999; 111:87-91.

4. Micci F, Teixeira MR, Scheistroen M, Abeler VM, Heim S. Cytogenetic characterization of tumors of the vulva and vagina. Genes ChromosomesCancer. 2003; 38:137-148.

5. Worsham MJ, Van Dyke DL, Grenman SE, Grenman R, Hopkins MP, Roberts JA, Gasser KM, Schwartz DR, Carey TE. Consistent chromosome abnormalities in squamous cell carcinoma of the vulva. Genes ChromosomesCancer. 1991; 3:420-432.

6. Agostini A, Panagopoulos I, Andersen HK, Johannesen LE, Davidson B, Trope CG, Heim S, Micci F. HMGA2 expression pattern and TERT mutations in tumors of the vulva. OncolRep. 2015:10.

7. Agostini A, Panagopoulos I, Andersen HK, Johannesen LE, Davidson B, Trope CG, Heim S, Micci F. HMGA2 expression pattern and TERT mutations in tumors of the vulva. Oncology reports. 2015; 33:2675-2680.

8. Kristjansdottir K, Fogarty EA, Grimson A. Systematic analysis of the Hmga2 3' UTR identifies many independent regulatory sequences and a novel interaction between distal sites. RNA. 2015; 21:1346-1360.

9. Wang YY, Ren T, Cai YY, He XY. MicroRNA let-7a inhibits the proliferation and invasion of nonsmall cell lung cancer cell line $95 \mathrm{D}$ by regulating K-Ras and HMGA2 gene expression. Cancer BiotherRadiopharm. 2013; 28:131-137.

10. Lin Y, Liu AY, Fan C, Zheng H, Li Y, Zhang C, Wu S, Yu D, Huang Z, Liu F, Luo Q, Yang CJ, Ouyang G. MicroRNA33b Inhibits Breast Cancer Metastasis by Targeting HMGA2, SALL4 and Twist1. SciRep. 2015; 5:9995. doi: 10.1038/srep09995.:9995.

11. Liu Y, Liang H, Jiang X. miR-1297 promotes apoptosis and inhibits the proliferation and invasion of hepatocellular carcinoma cells by targeting HMGA2. IntJMolMed. 2015:10

12. Wang T, Wang G, Hao D, Liu X, Wang D, Ning N, Li X. Aberrant regulation of the LIN28A/LIN28B and let-7 loop in human malignant tumors and its effects on the hallmarks of cancer. MolCancer. 2015; 14:125. doi: 10.1186/ s12943-015-0402-5.:125-0402.

13. Cleynen I, Van de Ven WJ. The HMGA proteins: a myriad of functions (Review). IntJOncol. 2008; 32:289-305.

14. Chiappetta G, Avantaggiato V, Visconti R, Fedele M, Battista S, Trapasso F, Merciai BM, Fidanza V, Giancotti V, Santoro M, Simeone A, Fusco A. High level expression of the HMGI (Y) gene during embryonic development. Oncogene. 1996; 13:2439-2446.
15. Rogalla P, Drechsler K, Frey G, Hennig Y, Helmke B, Bonk U, Bullerdiek J. HMGI-C expression patterns in human tissues. Implications for the genesis of frequent mesenchymal tumors. AmJPathol. 1996; 149:775-779.

16. Pallante P, Sepe R, Puca F, Fusco A. High Mobility Group A (HMGA) proteins as tumor markers. Frontiers in Medicine. 2015; 2.

17. Hetland TE, Holth A, Kaern J, Florenes VA, Trope CG, Davidson B. HMGA2 protein expression in ovarian serous carcinoma effusions, primary tumors, and solid metastases. Virchows Arch. 2012; 460:505-513.

18. Suh SS, Yoo JY, Cui R, Kaur B, Huebner K, Lee TK, Aqeilan RI, Croce CM. FHIT suppresses epithelialmesenchymal transition (EMT) and metastasis in lung cancer through modulation of microRNAs. PLoSGenet. 2014; 10:e1004652.

19. Schoenmakers EF, Wanschura S, Mols R, Bullerdiek J, Van den Berghe H, Van de Ven WJ. Recurrent rearrangements in the high mobility group protein gene, HMGI-C, in benign mesenchymal tumours. NatGenet. 1995; 10:436-444.

20. Park SM, Shell S, Radjabi AR, Schickel R, Feig C, Boyerinas B, Dinulescu DM, Lengyel E, Peter ME. Let-7 prevents early cancer progression by suppressing expression of the embryonic gene HMGA2. Cell Cycle. 2007; 6:2585-2590.

21. Kolenda T, Przybyla W, Teresiak A, Mackiewicz A, Lamperska KM. The mystery of let-7d - a small RNA with great power. ContempOncol(Pozn). 2014; 18:293-301.

22. Sterenczak KA, Eckardt A, Kampmann A, Willenbrock $\mathrm{S}$, Eberle N, Langer F, Kleinschmidt S, HewickerTrautwein M, Kreipe H, Nolte I, Murua EH, Gellrich NC. HMGA1 and HMGA2 expression and comparative analyses of HMGA2, Lin28 and let-7 miRNAs in oral squamous cell carcinoma. BMCCancer. 2014; 14:694. doi: 10.1186/1471-2407-14-694.:694-614.

23. Liu Q, Lv GD, Qin X, Gen YH, Zheng ST, Liu T, Lu XM. Role of microRNA let-7 and effect to HMGA2 in esophageal squamous cell carcinoma. MolBiolRep. 2012; 39:1239-1246.

24. Waters CE, Saldivar JC, Hosseini SA, Huebner K. The FHIT gene product: tumor suppressor and genome “caretaker". Cell MolLife Sci. 2014; 71:4577-4587.

25. Joannes A, Bonnomet A, Bindels S, Polette M, Gilles C, Burlet H, Cutrona J, Zahm JM, Birembaut P, NawrockiRaby B. Fhit regulates invasion of lung tumor cells. Oncogene. 2010; 29:1203-1213.

26. Morishita A, Zaidi MR, Mitoro A, Sankarasharma D, Szabolcs M, Okada Y, D'Armiento J, Chada K. HMGA2 is a driver of tumor metastasis. Cancer Res. 2013; 73:4289-4299.

27. Lamouille S, Xu J, Derynck R. Molecular mechanisms of epithelial-mesenchymal transition. NatRevMolCell Biol. 2014; 15:178-196. 